\title{
Alcohol and hepatitis virus-dysregulated IncRNAs as potential biomarkers for hepatocellular carcinoma
}

\author{
Hao Zheng ${ }^{1, *}$, Pinxue Li ${ }^{1, *}$, James G. Kwok ${ }^{1, *}$, Avinaash Korrapati ${ }^{1}$, Wei Tse $\mathbf{L i}^{1}$, \\ Yuanhao Qu ${ }^{1}$, Xiao Qi Wang ${ }^{2}$, Tatiana Kisseleva ${ }^{1}$, Jessica Wang-Rodriguez ${ }^{3}$ and \\ Weg M. Ongkeko ${ }^{1}$ \\ ${ }^{1}$ Department of Surgery, University of California, San Diego, La Jolla, California, USA \\ ${ }^{2}$ Department of Surgery, The University of Hong Kong, Pokfulam, Hong Kong, China \\ ${ }^{3}$ Veterans Administration Medical Center and Department of Pathology, University of California, San Diego, La Jolla, California, \\ USA \\ *These authors contributed equally to this work
}

Correspondence to: Weg M. Ongkeko, email: wongkeko@ucsd.edu

Keywords: InCRNA; hepatocellular carcinoma; alcohol; hepatitis

Received: August 06, $2017 \quad$ Accepted: November 09, $2017 \quad$ Published: December 05, 2017

Copyright: Zheng et al. This is an open-access article distributed under the terms of the Creative Commons Attribution License 3.0 (CC BY 3.0), which permits unrestricted use, distribution, and reproduction in any medium, provided the original author and source are credited.

\section{ABSTRACT}

Hepatocellular carcinoma (HCC) is one of the leading causes of cancerrelated deaths because of frequent late detection and poor therapeutic outcomes, necessitating the need to identify effective biomarkers for early diagnosis and new therapeutic targets for effective treatment. Long noncoding RNAs (IncRNAs) have emerged as promising molecular markers for diagnosis and treatment. Through analysis of patient samples from The Cancer Genome Atlas database, we identified putative IncRNAs dysregulated in HCC and by its risk factors, hepatitis infection and alcohol consumption. We identified 184 IncRNAs dysregulated in HCC tumors versus paired normal samples, 53 IncRNAs dysregulated in alcohol-drinking patients with hepatitis B, and 5, 456 IncRNAs dysregulated in patients with hepatitis infection. A panel of these candidate IncRNAs' expressions correlated significantly with patient survival, clinical variables, and known genomic alteration in HCC. Two most significantly dysregulated IncRNAs in our computational analysis, Inc-CFP-1:1 and Inc-CD164L2-1:1, were validated in vitro to be dysregulated by alcohol. Our findings suggest that IncRNAs dysregulated by different etiologies of HCC serve as potential disease markers and can be further investigated to develop personalized prevention, diagnosis, and treatment strategies.

\section{INTRODUCTION}

Hepatocellular carcinoma (HCC) is the most common class of liver cancer, accounting for $70-90 \%$ of primary liver cancer cases [1]. Because of limitations in diagnostic methods, HCC is often diagnosed late, when intrahepatic and extrahepatic metastasis are likely to have already occurred, leading to poor clinical outcome and therapeutic response $[1,2]$. HCC causes 750,000 death annually worldwide, the second highest total mortality out of all human cancers [3]. The five-year survival rate for HCC has remained below $20 \%$ [4]. Therefore, there is an urgent need for the discovery of biomarkers that can allow for early diagnosis of HCC and prediction of metastasis risk.

Long noncoding RNAs (lncRNAs) are noncoding RNAs over 200 bases in length that serve a variety of roles in regulating protein levels and gene expression [1]. LncRNAs have been extensively documented as important regulatory molecules involved in tumorigenesis [5]. A number of IncRNAs were revealed to have significant functions in the pathogenesis of HCC and could serve either tumor suppressing or oncogenic roles. Active pathological mechanisms in HCC such as the Wnt signaling pathway, the STAT3 signaling pathway, and 
epithelial-to-mesenchymal transition (EMT) were shown to be regulated by lncRNAs such as DANCR, ATB, and MALAT1, respectively [6-8]. Other lncRNAs involved in HCC include HOTAIR (HOX transcript antisense RNA) and HULC (Highly Upregulated in Liver Cancer), which are also both involved in multiple cancers beside HCC $[3,9]$. Because of their extensive involvement in tumorigenesis, lncRNAs are promising candidates as biomarkers for prediction of prognosis in HCC.

Important risk factors in the development of $\mathrm{HCC}$ are alcohol consumption and the hepatitis virus. An estimated half of HCC patients have an hepatitis B virus (HBV) infection, while thirty to forty percent of HBV patients ultimately develop HCC $[10,11]$. Hepatitis C virus (HCV) infection causes an estimated seventeen-fold increase in the risk of developing HCC compared to nonhepatitis individuals [12]. For people with heavy alcohol consumption, ten to thirty percent develop alcoholic steatohepatitis, while ten to twenty percent develop liver cirrhosis [13]. Both alcoholic steatohepatitis, a type of fatty liver disease, and liver cirrhosis can lead to development of HCC. Because HCC is a heterogeneous disease with various prognostic factors and prognostic outcomes, the risk factors involved in the development of $\mathrm{HCC}$ for specific patients must be considered in order to develop personalized diagnostic and treatment methods.

Most studies investigating lncRNAs involved in HCC were limited by the small size of patient cohorts and the lack of specific HCC etiology focus, with many studies comparing only tumor samples with normal samples [1]. While there have been studies exploring differential gene expression for coding genes in HCC caused by different risk facts, to the best of our knowledge, no study has compared the role of 1 ncRNAs in $\mathrm{HBV} / \mathrm{HCV}$ - related HCC to their role in alcohol-related HCC. We downloaded RNA-sequencing data for $222 \mathrm{HCC}$ patients from The Cancer Genome Atlas (TCGA) database to obtain a substantial cohort for analysis. We then analyzed lncRNA expression levels of patient normal samples versus that of three tumor sample cohorts, based on HBV infection, HCV infection, and history of alcohol consumption. Finally, the expressions of selected lncRNAs were verified in vitro.

\section{RESULTS}

\section{Identification of alcohol and hepatitis- dysregulated IncRNAs}

Clinical and RNA-sequencing data for $222 \mathrm{HCC}$ patients and 50 normal liver tissues were sorted into four cohorts based on their clinical history on viral hepatitis infection and alcohol consumption: (1) HBV+ $\mathrm{HCV}-$ drinker $(n=34)$; (2) HBV $+\mathrm{HCV}-$ non-drinker $(n=109)$; (3) HBV- HCV+ nondrinker $(n=32)$; and (4) $\mathrm{HBV}+\mathrm{HCV}+$ nondrinker $(n=47)$. To identify lncRNAs dysregulated by alcohol in the context of HBV, three differential expression analyses were performed using the Bioconductor package edgeR: (a) $\mathrm{HBV}+\mathrm{HCV}-$ drinker versus normal liver; (b) $\mathrm{HBV}+\mathrm{HCV}-$ non-drinker versus normal liver; and (c) $\mathrm{HBV}+\mathrm{HCV}-$ drinker versus $\mathrm{HBV}+$ $\mathrm{HCV}$ - non-drinker (Figure 1A). 53 lncRNA transcripts were found to be significantly dysregulated due to alcohol in the context of HBV (FDR < 0.05) (Figure 1B) and the top 10 most dysregulated lncRNAs due to alcohol use are shown in Table 1. To identify lncRNAs dysregulated by hepatitis virus, three differential expression analyses were performed: (d) $\mathrm{HBV}+\mathrm{HCV}$ - non-drinker versus normal; (e) HBV- $\mathrm{HCV}+$ non-drinker versus normal liver; and (f) $\mathrm{HBV}+\mathrm{HCV}+$ non-drinker versus normal liver. 12,328, 20,873 and 19,194 lncRNA transcripts were differentially expressed in patients with $\mathrm{HBV}$, $\mathrm{HCV}$, and both HBV and HCV, respectively, compared to normal liver. 5,456 lncRNA transcripts were found to be commonly dysregulated by $\mathrm{HBV}$ and $\mathrm{HCV}$ in all three comparisons above $($ FDR $<0.05)$ (Figure $1 C)$. The top 10 most dysregulated lncRNAs due to hepatitis virus are shown in Table 2. In addition, a pairwise differential expression analysis was performed on 50 tumor and adjacent normal pairs. $184 \operatorname{lncRNAs}$ were found to be significantly dysregulated (FDR $<0.05)$ in HCC tumors compared to adjacent normal, 32 of which were implicated in the previous alcohol and/or hepatitis analyses. The top 10 most dysregulated lncRNAs in tumor samples are shown in Table 3.

\section{Identification of dysregulated IncRNAs correlated to patient survival and other clinical outcomes}

To explore the clinical significance of lncRNAs identified in the differential expression analyses, we examined the relationship between their expression levels and overall patient survival. Modeling lncRNA expression as a binary variable (high/low), we found that relative high expression of five unregulated lncRNAs (lnc-FABP6-4:1, lnc-CALM2-2:5, lnc-CALM2-2:7, Inc-HPS3-2:3 and lnc-PKD2-2:2) and four downregulated lncRNAs (lncCD164L2-1:1, lnc-CFP-1:1, lnc-CLEC12B-1:1 and lncRP11.650K20.3.1.2.1) significantly associated with poor patient survival $(p<0.05)$ (Figure 2). Further, we correlated the expression of these genes to key clinical outcomes such as vital status, tumor grade and pathological tumor stage. Three lncRNAs (lnc-CLEC12B-1:1, lnc-HPS3-2:3 and lncRP11.650K20.3.1.2.1) significantly correlated with patient vital status $(p<0.05)$. Notably, stage-dependent correlation was observed between the expression level of two lncRNAs, lnc-FABP6-4:1 and lnc-CD164L2-1:1, and tumor grade, a grading system that characterizes level of differentiation of malignant cancer cells. Lastly, stage-dependent correlation was also found between lnc-CFP-1:1 and pathologic tumor stage, a staging system describing the development of liver cancer in terms of pathology. 


\section{Association of dysregulated IncRNAs with known HCC genomic alterations}

Numerous genomic alternations, including genetic mutations and DNA copy number variations, have been reported in HCC and have been associated with liver cancer development, progression and metastasis $[14,15]$. To explore the potential functions of lncRNAs in relationship to canonical HCC driver events, we utilized Wilcoxon rank-sum testing to identify correlations between lncRNA expression and tumor mutational status or copy number variation. We focused our analysis on the 10 most frequently mutated genes in HCCs, as determined by Debuire et al. [16]. We found nine lncRNAs to be significantly associated with mutation status $(p<0.05)$ (Figure 4$)$. Notably, four lncRNAs (lnc-RPRD2-3:1, lnc-KCNA5-3:4, lnc-CFP-1:1 and lnc-CD164L2-1:1) correlated strongly with the mutation status of IGF2R, a tumor suppressor commonly mutated in human liver and breast cancer. lnc-HPS3-2:3 expression significantly correlated with incidence of mutated CTNNB1 $(p=0.020)$, a well-studied protooncogene. Additionally, pairwise analysis between lncRNA expression and incidence of copy number variations revealed widespread correlations. lnc-FABP6-4:1, an lncRNA upregulated over 11 fold in HCC tumor compared to normal liver (Table 2), was found to have extensive correlation with copy number variations of 16 locations on chromosomes 4, 8, 9, 13, 14, 16, 17 and 19 (Figure 5A). lnc-AFM-2:1, another upregulated lncRNA in HCC patients (Table 1), correlated strongly with copy number variations of two locations on chromosome $8(p<0.01)$ (Figure 5B). Moreover, three lncRNAs, lnc-CD164L2-1:1, lnc-CFP-1:1

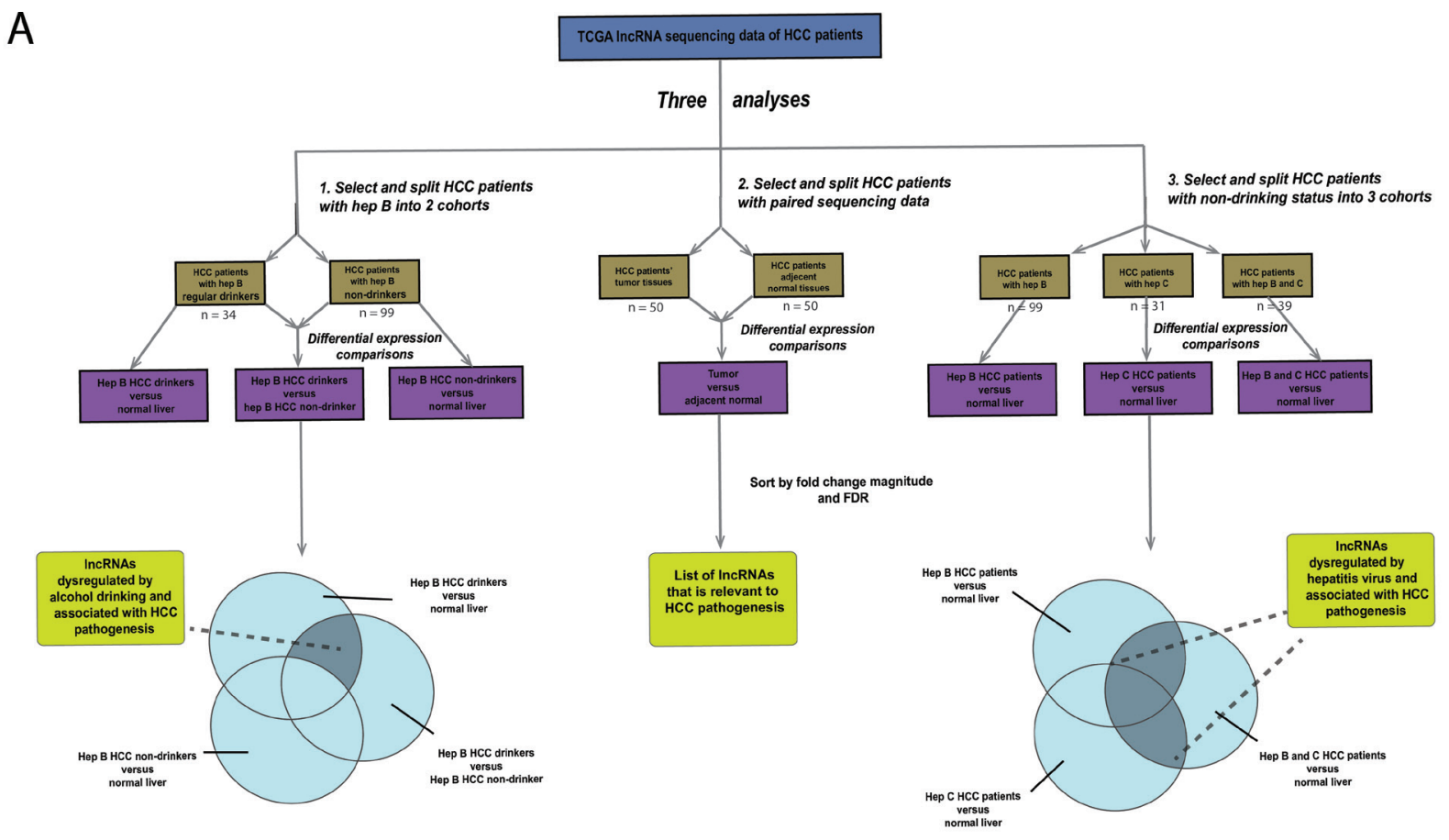

B

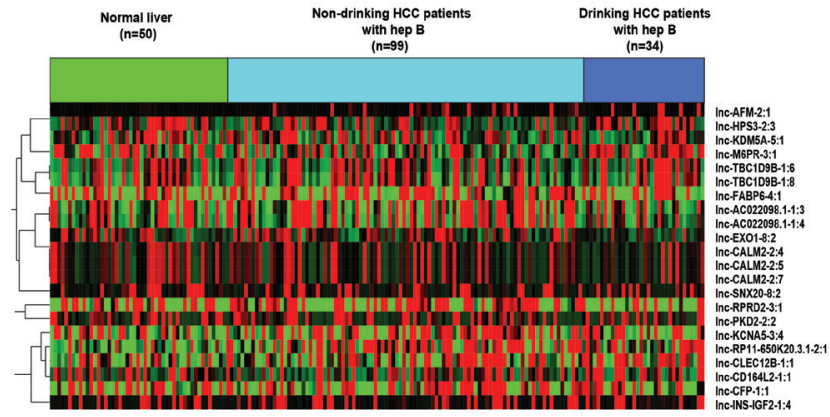

C

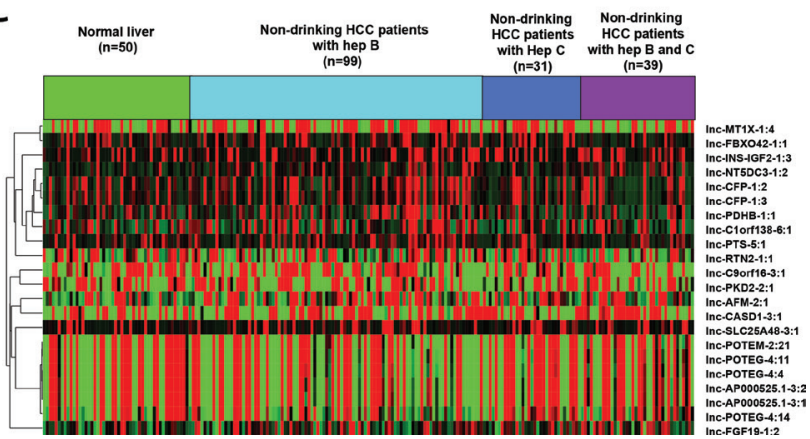

Figure 1: Identification of alcohol and hepatitis-dysregulated IncRNAs. (A) Schematic illustrating the analysis approach used to identify alcohol and hepatitis-dysregulated lncRNA candidates $(p<0.05$, FDR $<0.05)$. (B-C) Heatmap depicting normalized expression levels (in counts-per-million) of the 22 lncRNAs transcripts with the largest magnitude of dysregulation in (B) $\mathrm{HBV}+\mathrm{HCV}-\mathrm{drinker}$ versus $\mathrm{HBV}+\mathrm{HCV}-$ nondrinker and $(\mathrm{C}) \mathrm{HBV}+\mathrm{HCV}+$ nondrinker versus normal liver. 
Table 1: Significantly dysregulated IncRNAs in alcohol comparisons for $\mathrm{HBV}+$ patients

\begin{tabular}{lcccccccc}
\hline & \multicolumn{3}{c}{ HBV+ drinker versus HBV+ nondrinker } & \multicolumn{3}{c}{ HBV+ drinker versus normal liver } \\
\cline { 2 - 8 } & Fold change & logCPM & P value & FDR & Fold change & logCPM & P value & FDR \\
\hline Inc-AFM-2:1 & 4.80 & 8.74 & $1.3 \mathrm{E}-04$ & $3.3 \mathrm{E}-02$ & 120.55 & 8.62 & $1.1 \mathrm{E}-21$ & $5.3 \mathrm{E}-20$ \\
Inc-HPS3-2:3 & 4.29 & 1.96 & $1.6 \mathrm{E}-09$ & $3.9 \mathrm{E}-06$ & 2.27 & 2.48 & $9.1 \mathrm{E}-04$ & $2.0 \mathrm{E}-03$ \\
Inc-TBC1D9B-1:6 & 3.06 & 7.28 & $3.6 \mathrm{E}-07$ & $5.4 \mathrm{E}-04$ & 12.35 & 7.01 & $2.0 \mathrm{E}-25$ & $1.8 \mathrm{E}-23$ \\
Inc-TBC1D9B-1:8 & 2.74 & 7.89 & $2.0 \mathrm{E}-06$ & $2.1 \mathrm{E}-03$ & 9.31 & 7.59 & $1.4 \mathrm{E}-22$ & $8.0 \mathrm{E}-21$ \\
Inc-M6PR-3:1 & 2.26 & 2.88 & $1.9 \mathrm{E}-04$ & $4.1 \mathrm{E}-02$ & 6.53 & 2.44 & $3.7 \mathrm{E}-20$ & $1.4 \mathrm{E}-18$ \\
Inc-AC022098.1-1:3 & -2.50 & 3.59 & $1.0 \mathrm{E}-05$ & $6.4 \mathrm{E}-03$ & 1.76 & 1.93 & $4.7 \mathrm{E}-06$ & $1.6 \mathrm{E}-05$ \\
Inc-EXO1-8:2 & -2.58 & 1.79 & $3.0 \mathrm{E}-05$ & $1.4 \mathrm{E}-02$ & 1.33 & 0.31 & $1.5 \mathrm{E}-03$ & $3.2 \mathrm{E}-03$ \\
Inc-AC022098.1-1:4 & -2.77 & 3.23 & $1.9 \mathrm{E}-05$ & $9.4 \mathrm{E}-03$ & 1.90 & 1.39 & $4.5 \mathrm{E}-06$ & $1.5 \mathrm{E}-05$ \\
Inc-CALM2-2:4 & -2.90 & 2.85 & $4.8 \mathrm{E}-05$ & $1.7 \mathrm{E}-02$ & 1.48 & 1.11 & $5.7 \mathrm{E}-04$ & $1.3 \mathrm{E}-03$ \\
Inc-CALM2-2:5 & -3.00 & 2.83 & $3.5 \mathrm{E}-05$ & $1.5 \mathrm{E}-02$ & 1.67 & 0.97 & $1.7 \mathrm{E}-05$ & $5.2 \mathrm{E}-05$ \\
\hline
\end{tabular}

Table 2: Significantly dysregulated IncRNAs in hepatitis comparisons

\begin{tabular}{|c|c|c|c|c|c|c|c|c|c|c|c|c|}
\hline & \multicolumn{4}{|c|}{ HBV+ HCV- HCC versus normal liver } & \multicolumn{4}{|c|}{$\mathrm{HBV}-\mathrm{HCV}+\mathrm{HCC}$ versus normal liver } & \multicolumn{4}{|c|}{$\mathrm{HBV}+\mathrm{HCV}+\mathrm{HCC}$ versus normal liver } \\
\hline & $\begin{array}{c}\text { Fold } \\
\text { change }\end{array}$ & $\log \mathrm{CPM}$ & $P$ value & FDR & $\begin{array}{c}\text { Fold } \\
\text { change }\end{array}$ & $\log C P M$ & $P$ value & FDR & $\begin{array}{c}\text { Fold } \\
\text { change }\end{array}$ & $\log C P M$ & $P$ value & FDR \\
\hline Inc-AFM-2:1 & 20.14 & 6.75 & $4.49 \mathrm{E}-13$ & $2.01 \mathrm{E}-12$ & 5.09 & 4.39 & $3.56 \mathrm{E}-06$ & $1.25 \mathrm{E}-05$ & 17.73 & 6.24 & $4.79 \mathrm{E}-13$ & 4.95E-12 \\
\hline Inc-C9orf16-3:1 & 22.05 & 5.23 & $3.93 \mathrm{E}-21$ & $4.43 \mathrm{E}-20$ & 26.71 & 4.87 & $3.37 \mathrm{E}-23$ & $2.28 \mathrm{E}-21$ & 26.02 & 5.18 & $1.20 \mathrm{E}-21$ & $4.30 \mathrm{E}-20$ \\
\hline Inc-CASD1-3:1 & 40.16 & 7.23 & $4.67 \mathrm{E}-19$ & $4.08 \mathrm{E}-18$ & 23.03 & 5.79 & $3.96 \mathrm{E}-16$ & $8.37 \mathrm{E}-15$ & 36.30 & 6.77 & $1.11 \mathrm{E}-22$ & $4.51 \mathrm{E}-21$ \\
\hline Inc-FBXO42-1:1 & -4.70 & 3.05 & $5.63 \mathrm{E}-11$ & $2.00 \mathrm{E}-10$ & -4.34 & 3.76 & $1.92 \mathrm{E}-08$ & $1.07 \mathrm{E}-07$ & -13.40 & 3.57 & $1.56 \mathrm{E}-26$ & $1.05 \mathrm{E}-24$ \\
\hline Inc-INS-IGF2-1:3 & -6.76 & 3.50 & $1.11 \mathrm{E}-11$ & $4.25 \mathrm{E}-11$ & -6.59 & 4.20 & $1.00 \mathrm{E}-07$ & 4.89E-07 & -12.16 & 4.06 & $1.57 \mathrm{E}-14$ & $2.04 \mathrm{E}-13$ \\
\hline Inc-MT1X-1:4 & -5.29 & 8.47 & $2.64 \mathrm{E}-12$ & $1.08 \mathrm{E}-11$ & -3.63 & 9.26 & $2.22 \mathrm{E}-06$ & $8.15 \mathrm{E}-06$ & -13.09 & 9.05 & $5.67 \mathrm{E}-23$ & $2.37 \mathrm{E}-21$ \\
\hline Inc-NT5DC3-1:2 & -12.05 & 2.76 & $4.02 \mathrm{E}-87$ & $1.22 \mathrm{E}-82$ & -12.30 & 3.55 & $5.68 \mathrm{E}-32$ & $1.39 \mathrm{E}-29$ & -11.81 & 3.49 & $1.75 \mathrm{E}-38$ & $6.62 \mathrm{E}-36$ \\
\hline Inc-PKD2-2:1 & 22.55 & 5.48 & $1.02 \mathrm{E}-16$ & $6.84 \mathrm{E}-16$ & 13.79 & 4.17 & $1.59 \mathrm{E}-17$ & $4.17 \mathrm{E}-16$ & 20.68 & 5.05 & $1.52 \mathrm{E}-20$ & $4.68 \mathrm{E}-19$ \\
\hline Inc-POTЕM-2:21 & 13.95 & 3.80 & $1.12 \mathrm{E}-34$ & $6.20 \mathrm{E}-33$ & 13.03 & 3.13 & $7.15 \mathrm{E}-36$ & $2.83 \mathrm{E}-33$ & 16.44 & 3.78 & $6.59 \mathrm{E}-49$ & $1.51 \mathrm{E}-45$ \\
\hline Inc-SLC25A48-3:1 & -6.90 & 3.75 & $8.34 \mathrm{E}-14$ & $4.05 \mathrm{E}-13$ & -9.83 & 4.43 & $1.14 \mathrm{E}-15$ & $2.20 \mathrm{E}-14$ & -16.38 & 4.32 & $1.14 \mathrm{E}-22$ & 4.63E-21 \\
\hline
\end{tabular}

Table 3: Significantly dysregulated IncRNAs in paired tumor and adjacent normal analysis

\begin{tabular}{|c|c|c|c|c|}
\hline \multicolumn{5}{|c|}{ Pairwise tumor versus adjacent normal } \\
\hline & Fold change & $\log C P M$ & $P$ value & FDR \\
\hline Inc-AKR1B10-1:1 & 14.17 & 7.42 & $9.48 \mathrm{E}-10$ & $6.80 \mathrm{E}-09$ \\
\hline lnc-ASPM-1:4 & 14.10 & 2.13 & $2.04 \mathrm{E}-60$ & $3.85 \mathrm{E}-57$ \\
\hline lnc-ASPM-1:5 & 14.10 & 2.13 & $2.04 \mathrm{E}-60$ & $3.85 \mathrm{E}-57$ \\
\hline Inc-BEST4-1:1 & 11.69 & 2.68 & $5.71 \mathrm{E}-40$ & $2.46 \mathrm{E}-37$ \\
\hline Inc-FABP6-4:1 & 11.03 & 3.69 & $8.75 \mathrm{E}-27$ & $9.86 \mathrm{E}-25$ \\
\hline Inc-CXCL12-4:2 & -16.08 & 2.47 & $1.08 \mathrm{E}-40$ & $5.04 \mathrm{E}-38$ \\
\hline Inc-TARS2-1:2 & -16.10 & 2.18 & $1.94 \mathrm{E}-44$ & $1.36 \mathrm{E}-41$ \\
\hline Inc-SEC22C-1:4 & -16.15 & 1.82 & $6.77 \mathrm{E}-41$ & $3.24 \mathrm{E}-38$ \\
\hline Inc-EIF2AK3-4:9 & -16.25 & 1.68 & $5.69 \mathrm{E}-15$ & $1.02 \mathrm{E}-13$ \\
\hline Inc-SEC22C-1:12 & -16.27 & 1.53 & $1.75 \mathrm{E}-41$ & $9.11 \mathrm{E}-39$ \\
\hline
\end{tabular}


and lnc-CLEC12B-1:1, all downregulated lncRNAs in tumor (Figure $1 \mathrm{~B}$ and $1 \mathrm{C}$ ), significantly correlated with the copy number variations of multiple locations on chromosomes 2, 11, 17, and 18 (Figure 5C-5E).

\section{In vitro validation of alcohol-dysregulated IncRNAs in liver cell lines}

We chose two lncRNAs, lnc-CFP-1:1 and lncCD164L2-1:1, for further in vitro validation for their strong correlations with both clinical outcomes and genomic alterations (Figures 2-5). Both of these lncRNAs are observed to be consistently downregulated in drinker HCC patients compared to normal livers (Figure 6A), with clusters of drinker HCC patients observed at low expressions for both lncRNAs (Figure 6B). lncCFP-1:1, a 569-nt transcript on chromosome X, and lncCD164L2-1:1, a 1,000-nt transcript on chromosome 1, have not been previously characterized. To validate their dysregulation due to alcohol, we treated the non-cancerous liver cell line L02 as well as the human hepatoma cell line Hep3B with $0.1 \%$ (17 mM), 0.3\% (34 mM) and $1 \%(170 \mathrm{mM})$ ethanol for 7 days. Upon treatment with ethanol, both lncRNAs were observed to be significantly downregulated, in a dose-dependent fashion, in both cell lines, with lnc-CFP-1:1 reduced to less than $20 \%$ of its original expression at $170 \mathrm{mM}$ alcohol.

\section{DISCUSSION}

We investigated lncRNAs deregulated in alcoholrelated $\mathrm{HCC}$ and $\mathrm{HBV} / \mathrm{HCV}$-related $\mathrm{HCC}$ to explore unique disease markers for different etiologies. lncRNAs have been documented as important functional molecules in the development of HBV/HCV-related HCC [17]. Our study identified 12,328 differentially expressed lncRNAs between $\mathrm{HBV}$-infected patient samples and normal samples. 20,873 lncRNAs were differentially expressed between HCV positive samples and normal samples. 19, 194 lncRNAs were differentially expressed between samples positive for both HBV and HCV and normal samples. The 5,456 lncRNAs that displayed significant
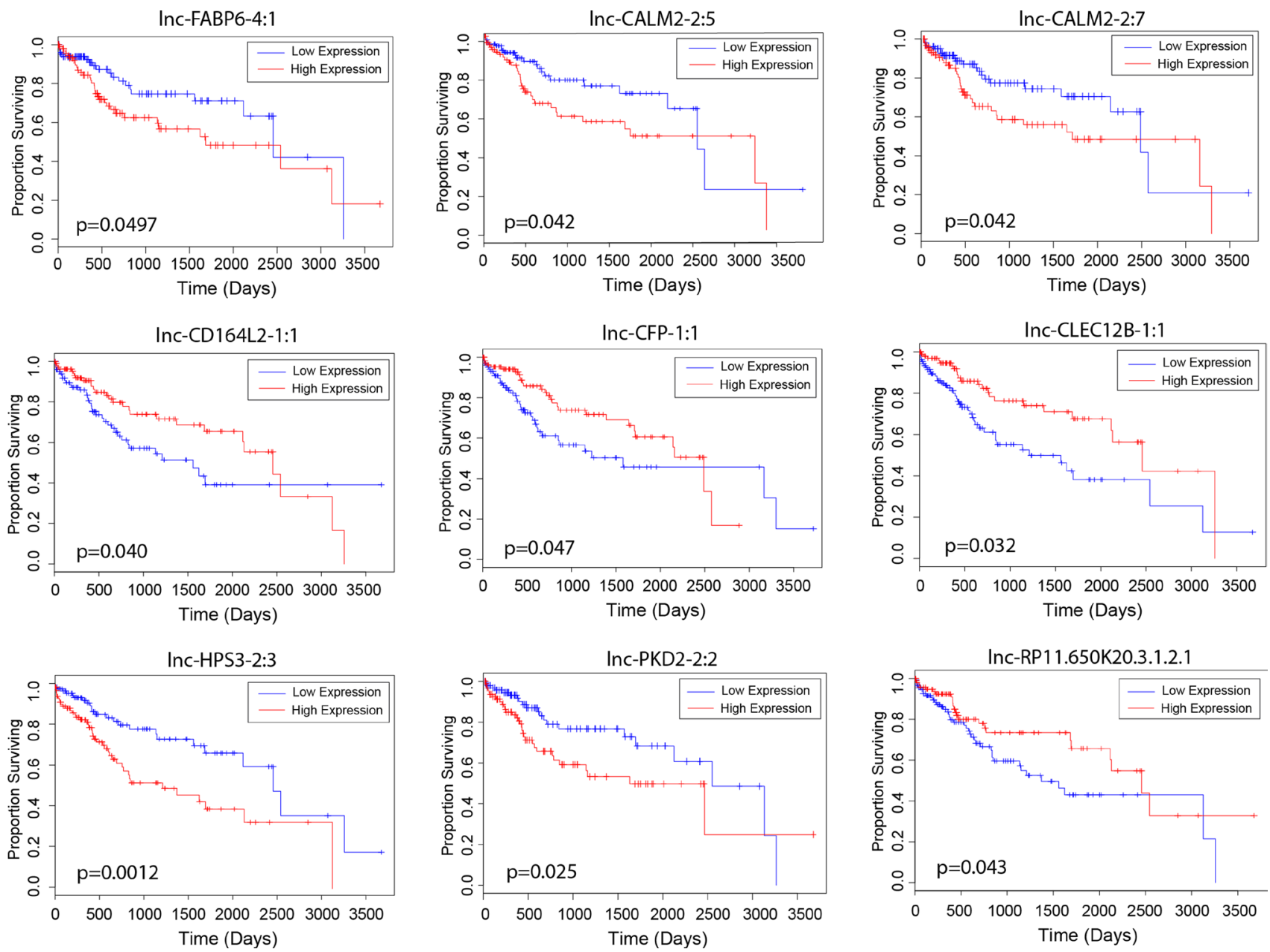

Figure 2: Association of IncRNA expression with patient survival. Kaplan-Meier curves depicting survival outcomes based on relative high and low expression of candidate lncRNAs proposed to be dysregulated by alcohol and/or viral hepatitis (Kaplan-Meier, $p<0.05)$. 
differential expression in all three permutations above were most likely to be highly involved in hepatitis induction of HCC. Despite the large numbers of lncRNAs that are deregulated in patients with $\mathrm{HBV} / \mathrm{HCV}$-related HCC, only the mechanisms of a select few lncRNAs have been extensively studied [6,17-20]. None of the most significantly dysregulated lncRNAs we found has been previously studied in $\mathrm{HCC}$, suggesting that our current picture of lncRNA dysregulation in HCC is incomplete.

From our analysis, 53 lncRNAs were associated with alcohol-related HCC. We found no previous study that explored the relationship between lncRNAs and alcohol consumption in HCC. The reason may be that alcohol intake often indirectly leads to genetic dysregulation- through causing elevated acetaldehyde levels, accumulation of iron, chronic liver inflammation, or liver fibrosis/cirrhosis-while hepatitis viruses integrate viral DNA directly into the host genome to cause dysregulation of genetic mechanisms [21, 22]. For example, alcohol consumption can lead to liver fibrosis, which can progress to liver cirrhosis. Cirrhotic livers led to HCC in $90 \%$ of HCC cases [23]. Knowledge of dysregulated lncRNAs in alcohol-related HCC may lead to development of prevention or treatment strategies for alcoholic cirrhosis, thereby arresting disease progression to HCC.
Candidate lncRNAs from our analysis were correlated with patient survival and clinical variables. The expression of four lncRNAs differentially expressed in HCC versus normal samples- lnc-FABP6-4:1, lnc-CD164L2-1:1, lncCFP-1:1, and lnc-CLEC12B-1:1- correlated with survival rates in the direction of dysregulation. The expression of three IncRNAs dysregulated by alcohol drinking- lncCALM2-2:5, lnc-CALM2-2:7, and lnc-HPS3-2:3- also correlated with patient survival. For lncRNAs dysregulated by hepatitis, lnc-PKD2-2:2 and lnc-RP11-650K20.3.1-2:1 expressions correlated with patient survival. The expression of the majority of these lncRNAs also correlated with vital status, tumor grade, or pathological tumor stage.

We correlated the expression of survival-related lncRNAs with mutation status and copy number variation to gain an understanding of their putative involvement in dysregulated genetic pathways in HCC. Inc-FABP6-4:1 upregulation correlated with presence of TP53 and RB1 mutation. IGF2R mutation correlated with downregulation of lnc-CFP-1:1 and lnc-CD164L2-1:1. Lnc-CD164L2 has a gene locus resides in the intronic region of the protein coding gene CD164, which has been found to act as a metastasis promoter in prostate cancer [24]. CTNNB1 mutation correlated with upregulation of lnc-HPS3-2:3. TP53, a tumor suppressor gene, is the most commonly mutated gene in cancer, including in $\mathrm{HCC}$; while
A

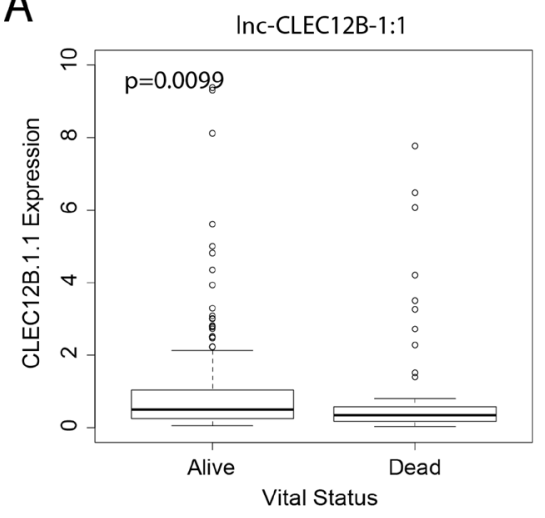

B

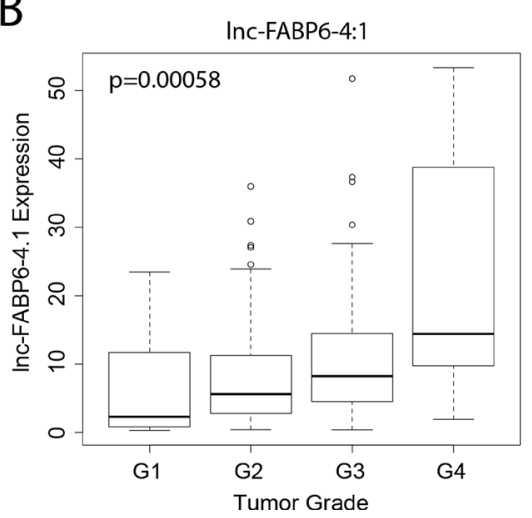

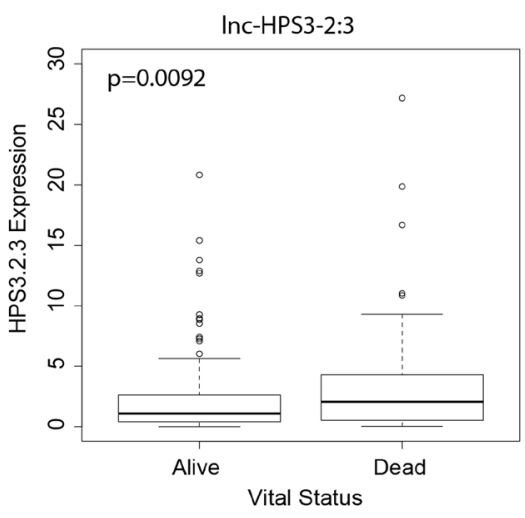

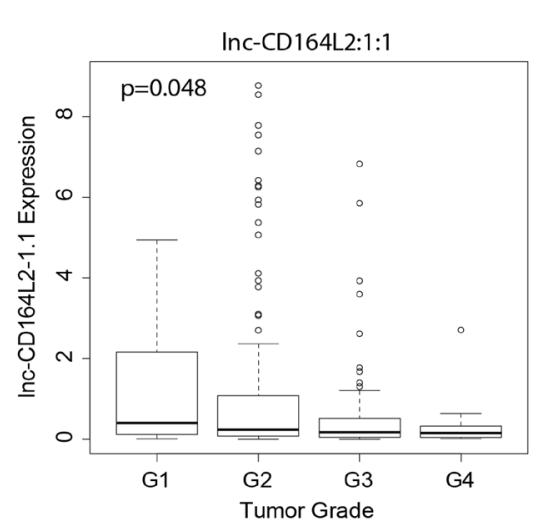

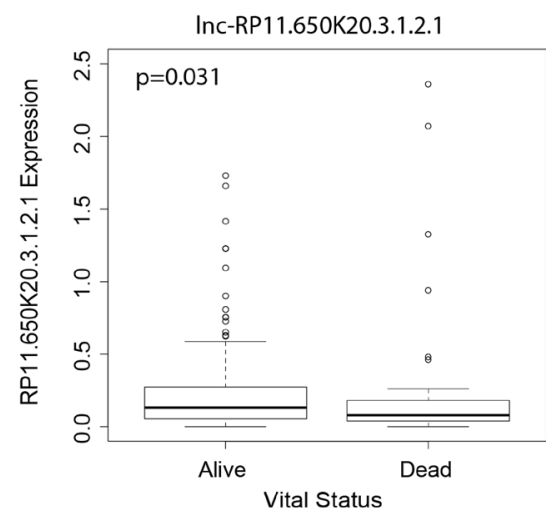

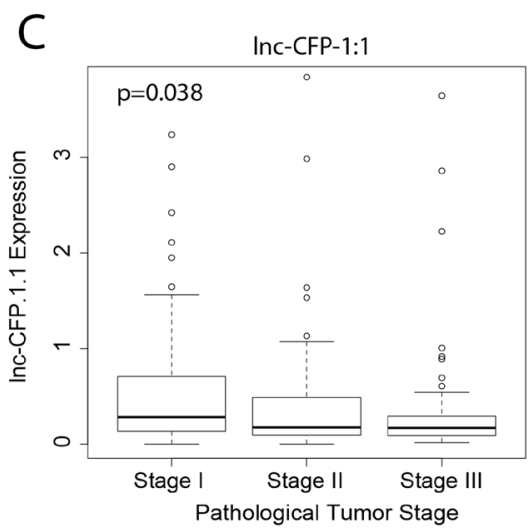

Figure 3: Association of IncRNA expression with clinical outcomes. Boxplots correlating expression of lncRNAs (counts-permillion) to (A) vital status, $(\mathbf{B})$ tumor grade, and $(\mathbf{C})$ pathologic tumor stage (Wilcoxon rank sum, $p<0.05$ ). 
CTNNB1 is the most commonly mutated proto-oncogene in HCC [25]. CTNNB1 is an integral element of the Wnt signaling pathway, which is activated in HCC and shown to be partly regulated by lncRNAs, such as lnc-DANCR [6]. RB1 is part of the RB1 tumor suppressing pathway, which is commonly inactivated in many cancers, including HCC [26]. IGF2R is also a tumor suppressor involved in multiple cancers and functions in HCC by inhibiting liver cell invasion [27].

To validate our correlations, we treated cells to different concentrations of alcohol in vitro and measured expression levels of lnc-CD164L2-1:1 and lnc-CFP-1:1, which correlated very well with patient survival and clinical variable. The decrease in their expression as alcohol concentration increases in both HCC and normal cell lines matches the direction of dysregulation in our statistical analysis and validates our hypothesis that alcohol dysregulates lncRNA expression.

No previous study, to the best of our knowledge, has explored the mechanism of possible synergism between the risk factors of hepatitis infection and alcohol intake. Studies from Italy, Taiwan, Japan, and the United States have found increased risk of HCC development when patients with $\mathrm{HCV}$ and $\mathrm{HBV}$ infections regularly drink alcohol, compared to non-drinking patients with hepatitis [28-31]. Further investigation of the mechanisms of
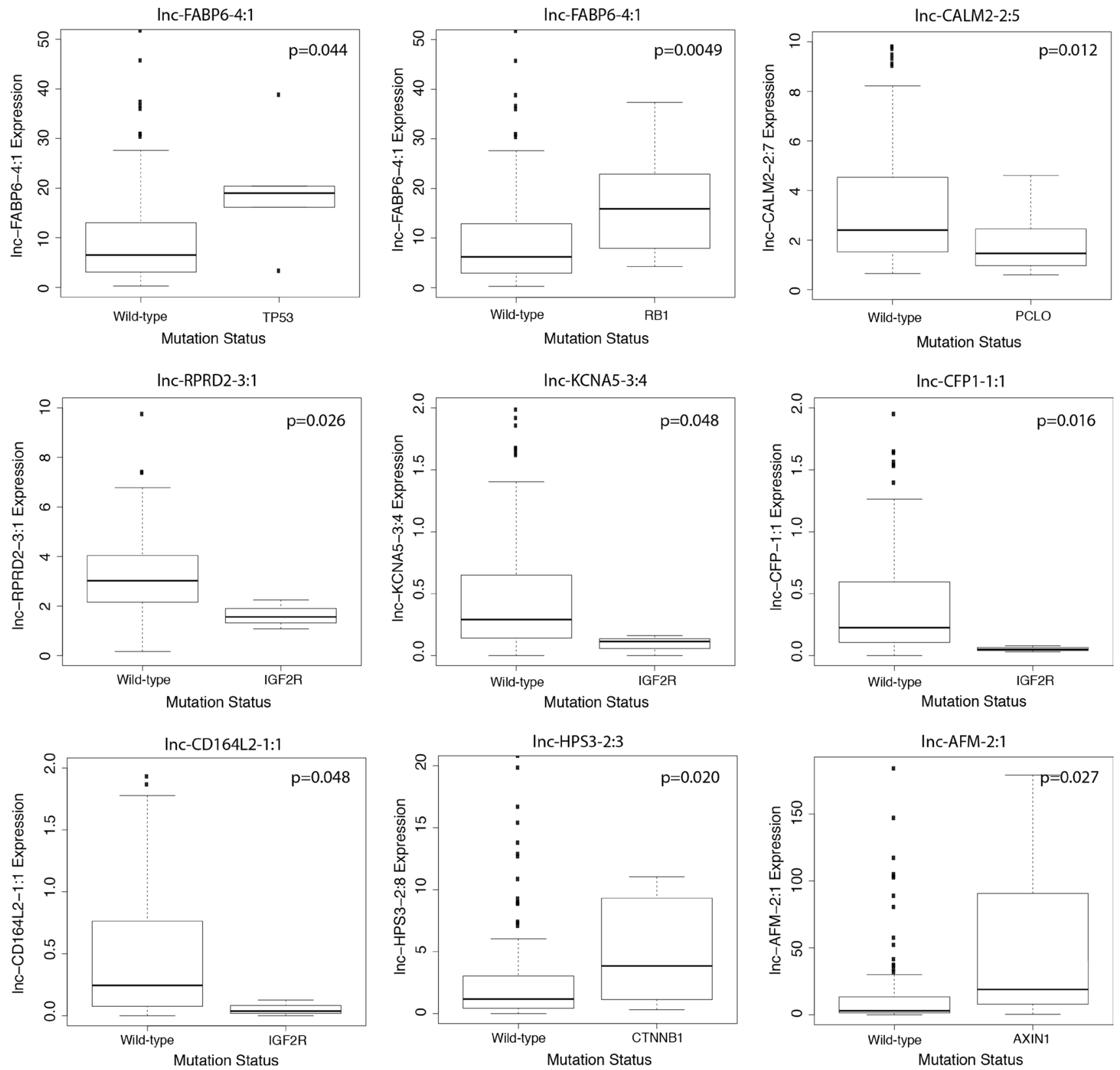

Figure 4: Correlation of IncRNA expression with gene mutations. Boxplot showing significant correlation in expression level of lncRNAs (counts-per-million) and mutation status of frequently mutated genes in HCCs (Wilcoxon rank sum, $p<0.05$ ). 
lncRNAs we identified to be involved in alcohol-related $\mathrm{HCC}$ and $\mathrm{HBV} / \mathrm{HCV}$-related $\mathrm{HCC}$ may provide insight into this mechanism of synergism, knowledge that can lead to better identification of high-risk individuals and provide focus for development of preventative methods.

The early diagnosis of hepatocellular carcinoma is critical to more effective treatment and better prognosis since the few current treatment options for potentially curing HCC, such as liver transplantation, ablation, or resection, are effective only for the early-stages of HCC [32]. Ultrasonography can be used for early diagnosis but only has a sensitivity of $60-80 \%$, despite a specificity of 94\% [33]. Biomarkers such as serum alpha-fetoprotein (AFP) and des-gamma carboxyprothrombin (DCP) failed as useful diagnostic tools because of similar sensitivity as ultrasonography [1]. In contrast, studies utilizing combinations of circulating lncRNAs or lncRNA with other types of RNAs as diagnostic biomarkers for HCC achieved sensitivities of over 90\% [34-37]. These studies suggested the usefulness of using lncRNAs as diagnostic markers, although the great majority of these studies investigated HCC cohort versus healthy liver cohort instead of taking specific risk factors into account [1]. Because different etiologies of $\mathrm{HCC}$ can result in dysregulation of different lncRNAs and disparate prognoses, our analysis of lncRNAs dysregulated in HCC induced by different risk factors can provide useful information about biomarkers present at the early stages of HCC.
Besides serving as diagnostic markers, lncRNAs can potentially be directly involved in the treatment of HCC. Direct delivery of tumor suppressing lncRNAs into liver cells or reducing expression of oncogenic lncRNAs through siRNA interference can be potential treatment strategies [1]. These options provide alternatives to the aforementioned invasive procedures for curing HCC. Furthermore, current treatment practices for HCC only consider the stage of disease, not its molecular etiology [38]. The use of lncRNAs for treatment may also lead to more personalized treatments of $\mathrm{HCC}$ for better therapeutic response.

\section{MATERIALS AND METHODS}

\section{RNA-sequencing datasets and clinical data}

RNA-sequencing datasets and clinical data for HCC patients and tumor-adjacent normal liver tissues were obtained on 8 July 2016 from The Cancer Genome Atlas (TCGA) (https://tcga-data.nci.nih.gov/tcga). 222 HCC patients and 50 tumor-adjacent normal liver tissues were retained for further analysis. These patients were sorted into four cohorts based on their clinical history on viral hepatitis infection and alcohol consumption: (1) HBV+ $\mathrm{HCV}$ - drinker $(n=34)$; (2) HBV+ HCV- non-drinker $(n=109)$; (3) HBV- HCV+ nondrinker $(n=32)$; and (4) $\mathrm{HBV}+\mathrm{HCV}+$ nondrinker $(n=47)$.
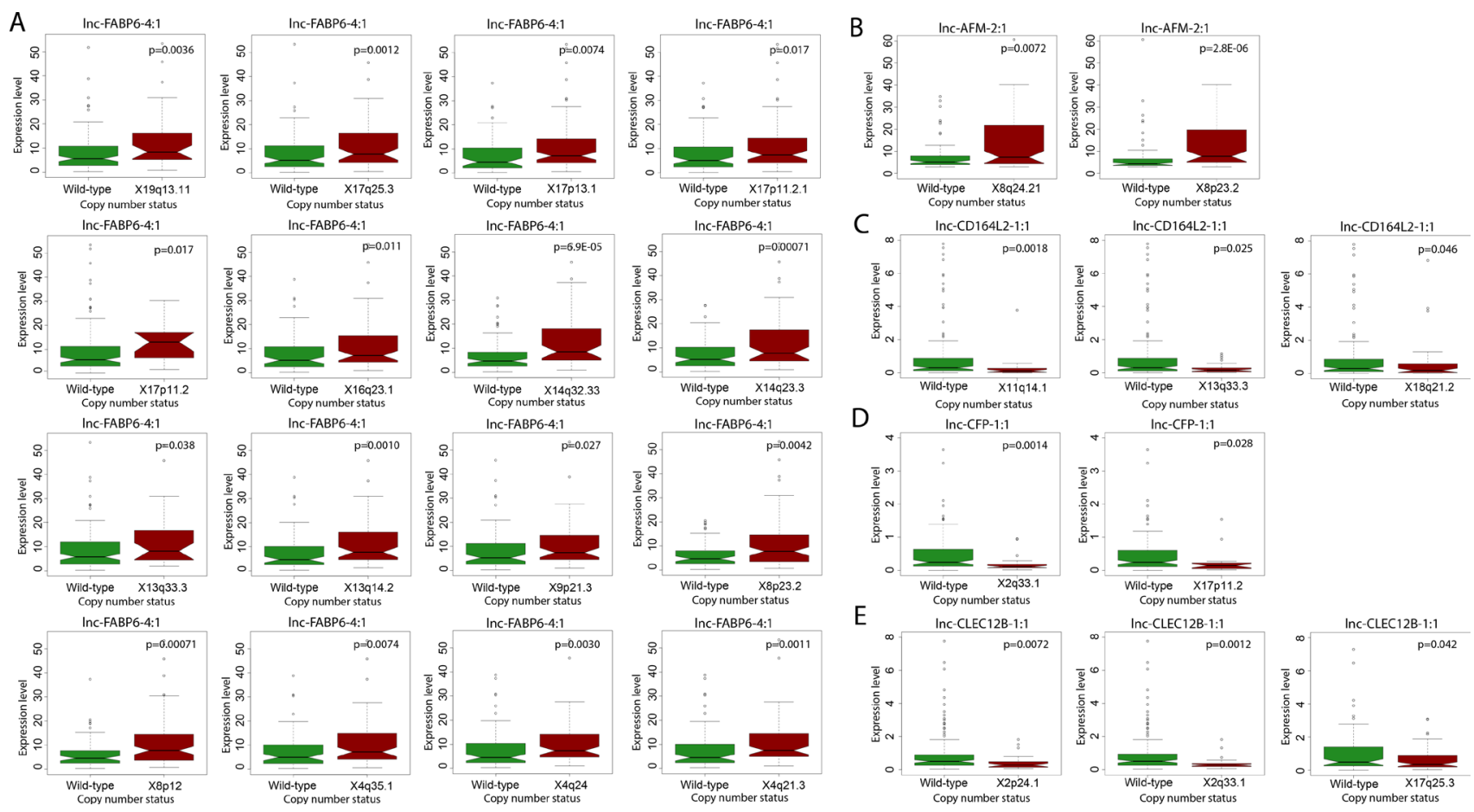

Figure 5: Correlation of IncRNA expression with copy number aberrations. Boxplot showing significant correlation in copy number variation status and expression level (counts-per-million) of (A) lnc-FABP6-4:1, (B) lnc-AFM-2:1, (C) lnc-CD164L2-1:1, (D) lnc-CFP-1:1, and (E) lnc-CLEC12B-1:1 (Wilcoxon rank sum, $p<0.05$ ). 


\section{IncRNA differential expression analyses}

lncRNA read counts were generated from RNAsequencing datasets via BEDtools coverageBed (https:// github.com/arq5x/bedtools2) [39] using lncRNA annotation files obtained from LNCipedia 3.0 (http://lncipedia.org/) [40], a database curating 113,438 lncRNA transcripts from sources including the Broad Institute, Ensembl, Gencode, Refseq, and NONCODE. The read count tables were imported into edgeR v3.0 (http://www.bioconductor. org/packages/release/bioc/html/edgeR.html) [41], and lowly expressed lncRNAs (counts-per-million $<1$ in more than one-half of samples) were filtered from the analysis. Following TMM normalization, pairwise designs were applied to identify significantly differentially expressed lncRNAs in (1) HBV+ HCV - drinker versus normal liver; (2) $\mathrm{HBV}+\mathrm{HCV}-$ non-drinker versus normal liver; (3) $\mathrm{HBV}+\mathrm{HCV}-$ drinker versus $\mathrm{HBV}+\mathrm{HCV}$ - non-drinker; (4) $\mathrm{HBV}-\mathrm{HCV}+$ non-drinker versus normal liver; and (5) $\mathrm{HBV}+\mathrm{HCV}+$ non-drinker versus normal liver.

\section{Association of IncRNA expression with patient survival and other clinical outcomes}

Survival analyses were performed using the KaplanMeier Model, with lncRNA expression in HCC tumors designated as a binary variable based on expression above or below the median. One patient with no clinical information was removed and $221 \mathrm{HCC}$ patients were retained for the analysis. Employing the Kruskal-Wallis test and lncRNA expression values (counts-per-million), we investigated lncRNA association patient vital status, tumor grade and pathologic stages. HCC patients in all four cohorts were used in this analysis.

\section{Association of IncRNA expression with tumor mutations and copy number aberrations}

Mutation calls for the HCC tumors were obtained from mutation annotation files (maf) generated by the Broad Institute GDAC Firehose on 5 September 2016.
A

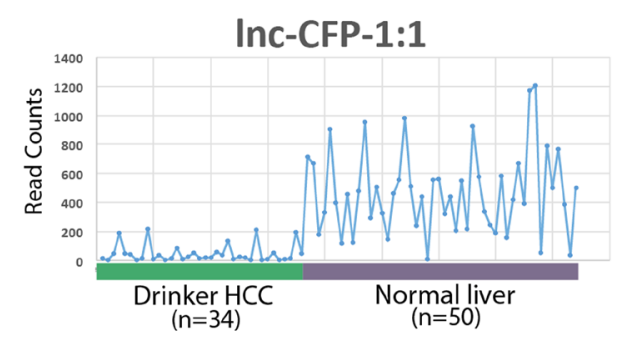

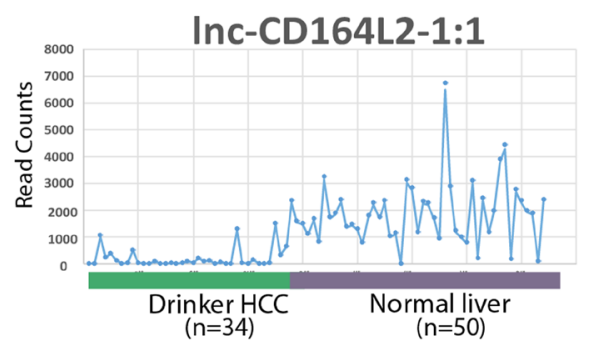

\section{B}

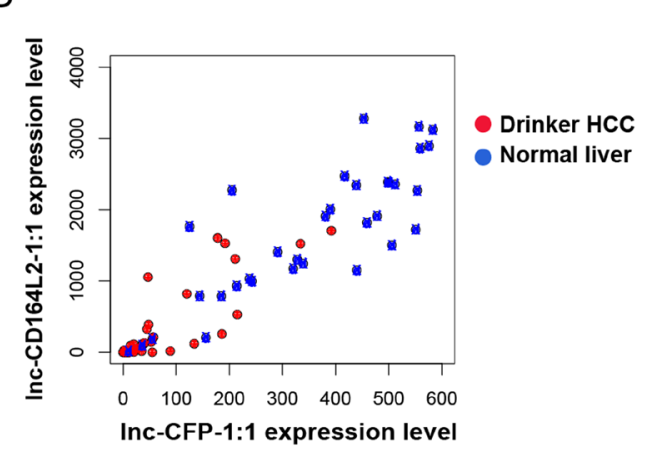

C

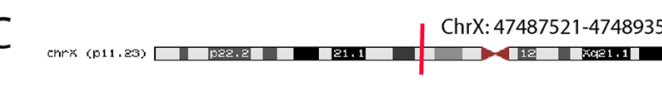

\begin{tabular}{l|l} 
Chr1:27700021-27701296 &
\end{tabular}

Figure 6: In vitro validation of alcohol-dysregulated IncRNAs in liver cell lines. (A) Expression plot of lnc-CFP-1:1 and lnc-CD164L2-1:1 in HCC drinkers compared to normal liver tissues. (B) Scatter plots showing clusters of HCC drinkers and normal liver tissues with respect to expression levels of lnc-CFP-1:1 and lnc-CD164L2-1:1. (C) Loci of lnc-CFP-1:1 and lnc-CD164L2-1:1. (D) qRTPCR verifies that $0.1 \%, 0.3 \%$ and $1 \%$ alcohol treatment downregulates lnc-CFP-1:1 and lnc-CD164L2-1:1 in L02 and Hep3B. All bar graphs are presented as the mean and error bars representing standard deviations. 
We focused our analysis on the 10 most frequently mutated genes in HCCs, as determined by Debuire et al [16]. Wilcoxon rank sum tests were employed to test for significant associations between lncRNA expression level (counts-per-million) and mutational status. Copy number variations for the TCGA tumors were obtained from the GISTIC2 pipeline in Broad GDAC Firehose on 6 July 2017. All significant (99\% confidence) focal amplifications and deletions were analyzed for correlation to lncRNA expression level using Wilcoxon rank sum tests, followed by Benjamini-Hochberg correction of lncRNA $p$-values.

\section{Cell culture and treatments with ethanol}

The non-cancerous liver cell line L02 and the human hepatoma cell line Hep3B were gifts from the Wang lab at University of Hong Kong. The cells were cultured in DMEM supplemented with $10 \%$ fetal bovine serum, $2 \%$ penicillin/streptomycin, and 2\% L-glutamate (GIBCO) and maintained at $37^{\circ} \mathrm{C}$ in a humidified $5 \% \mathrm{CO} 2 / 95 \%$ air atmosphere. These cells were exposed to ethanol for 7 days. The doses used for ethanol treatment were $0.1 \%, 0.3 \%$, and $1 \%$ by volume (approximate concentrations $17 \mathrm{mM}$, $51 \mathrm{mM}$, and $170 \mathrm{mM}$, respectively). We chose the $0.1 \%$ $(17 \mathrm{mM})$ dose to represent social drinking habits, as $0.1 \%$ is the blood alcohol level constituting legal intoxication in the U.S. [42]. The $0.3 \%$ (51 mM) ethanol dose was used to simulate binge drinking habits, as it is representative of the blood alcohol levels of moderate to heavy drinkers [43]. The $1 \%(170 \mathrm{mM})$ ethanol dose, while potentially lethal in humans, was employed as an upper limit control. Treatment media was replaced every 24 hours with fresh media containing the stated ethanol concentration. The tissue culture plates were sealed with paraffin film to reduce evaporative loss of ethanol from the media.

\section{RNA isolation and cDNA synthesis}

Upon completion of alcohol treatments, cells were harvested, and total cell lysates were collected. RNA was extracted using SurePrep RNA Isolation kit (Thermo Fisher Scientific, Inc.). Complementary DNA was synthesized according to the manufacturer's protocol, using LncProfiler qPCR Array kit (catalogue no. RA900A-1; System Biosciences, Mountain View, CA, USA).

\section{qRT-PCR}

qRT-PCRs were performed using FastStart Universal SYBR Green Master Mix (Roche Diagnostics) and run on a StepOnePlus Real-Time PCR System (Applied Biosystems). Results were analyzed using the $\Delta \Delta \mathrm{Ct}$ method and normalized to GAPDH expression. All primers were obtained from Bioneer and sequences are: GAACCTACT CTGTGCCAGCTC (lnc-CD164L2-1 forward), TTAC TCACCTGGCTCACCCT (lnc-CD164L2-1 reverse),
ACCCCACGAAGGTAGAAGC (LNC-CFP-1:1 forward) and GAGGTTTCCACCCAATCCCA (LNC-CFP-1:1 reverse).

\section{CONFLICTS OF INTEREST}

The authors declare that they have no conflicts of interest.

\section{FUNDING}

This work was supported by funding from the National Institutes of Health, grant number DE023242 to W.M.O.

\section{REFERENCES}

1. Klingenberg M, Matsuda A, Diederichs S, Patel T. Noncoding RNA in hepatocellular carcinoma: Mechanisms, biomarkers and therapeutic targets. J Hepatol. 2017; 67:603-18. https://doi.org/10.1016/j.jhep.2017.04.009.

2. Aldrighetti L, Pulitano C, Catena M, Arru M, Guzzetti E, Halliday J, Ferla G. Liver resection with portal vein thrombectomy for hepatocellular carcinoma with vascular invasion. Ann Surg Oncol. 2009; 16:1254. https://doi. org/10.1245/s10434-009-0383-y.

3. Qu Z, Yuan CH, Yin CQ, Guan Q, Chen H, Wang FB. Metaanalysis of the prognostic value of abnormally expressed lncRNAs in hepatocellular carcinoma. Onco Targets Ther. 2016; 9:5143-52. https://doi.org/10.2147/OTT.S108599.

4. Howlader AN, Krapcho M, Garshell J, Miller D, Altekruse S, Kosary C, Yu M, Ruhl J, Tatalovich Z, Mariotto A, Lewis D, Chen H, Feuer E, et al. SEER Cancer Statistics Review. 2014; 1975-2011. (SEER Cancer Statistics Review: National Cancer Insititute, Bethesda, MD).

5. Tsai MC, Spitale RC, Chang HY. Long intergenic noncoding RNAs: new links in cancer progression. Cancer Res. 2011; 71:3-7. https://doi.org/10.1158/0008-5472.CAN-10-2483.

6. Yuan SX, Wang J, Yang F, Tao QF, Zhang J, Wang LL, Yang Y, Liu H, Wang ZG, Xu QG, Fan J, Liu L, Sun SH, et al. Long noncoding RNA DANCR increases stemness features of hepatocellular carcinoma by derepression of CTNNB1. Hepatology. 2016; 63:499-511. https://doi. org/10.1002/hep.27893.

7. Yuan JH, Yang F, Wang F, Ma JZ, Guo YJ, Tao QF, Liu F, Pan W, Wang TT, Zhou CC, Wang SB, Wang YZ, Yang Y, et al. A long noncoding RNA activated by TGF-beta promotes the invasion-metastasis cascade in hepatocellular carcinoma. Cancer Cell. 2014; 25:666-81. https://doi. org/10.1016/j.ccr.2014.03.010.

8. Gutschner T, Hammerle M, Eissmann M, Hsu J, Kim Y, Hung G, Revenko A, Arun G, Stentrup M, Gross M, Zornig M, MacLeod AR, Spector DL, et al. The noncoding RNA MALAT1 is a critical regulator of the metastasis 
phenotype of lung cancer cells. Cancer Res. 2013; 73:1180 9. https://doi.org/10.1158/0008-5472.CAN-12-2850.

9. Yang Y, Chen L, Gu J, Zhang H, Yuan J, Lian Q, Lv G, Wang S, Wu Y, Yang YT, Wang D, Liu Y, Tang J, et al. Recurrently deregulated lncRNAs in hepatocellular carcinoma. Nat Commun. 2017; 8:14421. https://doi.org/10.1038/ ncomms14421.

10. Li Z, Zhao X, Jiang P, Xiao S, Wu G, Chen K, Zhang X, Liu H, Han X, Wang S, Li X. HBV is a risk factor for poor patient prognosis after curative resection of hepatocellular carcinoma: A retrospective case-control study. Medicine (Baltimore). 2016; 95:e4224. https://doi.org/10.1097/ MD.0000000000004224.

11. Yu TT, Xu XM, Hu Y, Deng JJ, Ge W, Han NN, Zhang MX. Long noncoding RNAs in hepatitis B virus-related hepatocellular carcinoma. World J Gastroenterol. 2015; 21:7208-17. https://doi.org/10.3748/wjg.v21.i23.7208.

12. Bartosch B, Thimme R, Blum HE, Zoulim F. Hepatitis C virus-induced hepatocarcinogenesis. J Hepatol. 2009; 51:810-20. https://doi.org/10.1016/j.jhep.2009.05.008.

13. Morgan TR, Mandayam S, Jamal MM. Alcohol and hepatocellular carcinoma. Gastroenterology. 2004; 127:S87-96.

14. Wang E. Understanding genomic alterations in cancer genomes using an integrative network approach. Cancer Lett. 2013; 340:261-9. https://doi.org/10.1016/j.canlet.2012.11.050.

15. Nishida N, Goel A. Genetic and epigenetic signatures in human hepatocellular carcinoma: a systematic review. Curr Genomics. 2011; 12:130-7. https://doi.org/10.2174/ 138920211795564359.

16. Lemoine A, Azoulay D, Jezequel-Cuer M, Debuire B. [Hepatocellular carcinoma]. Pathol Biol (Paris). 1999; 47:903-10.

17. Iizuka N, Oka M, Yamada-Okabe H, Mori N, Tamesa T, Okada T, Takemoto N, Tangoku A, Hamada K, Nakayama H, Miyamoto T, Uchimura S, Hamamoto Y. Comparison of gene expression profiles between hepatitis $B$ virus- and hepatitis C virus-infected hepatocellular carcinoma by oligonucleotide microarray data on the basis of a supervised learning method. Cancer Res. 2002; 62:3939-44.

18. Huang JF, Guo YJ, Zhao CX, Yuan SX, Wang Y, Tang GN, Zhou WP, Sun SH. Hepatitis B virus X protein $(\mathrm{HBx})$ related long noncoding RNA (lncRNA) down-regulated expression by HBx (Dreh) inhibits hepatocellular carcinoma metastasis by targeting the intermediate filament protein vimentin. Hepatology. 2013; 57:1882-92. https://doi. org/10.1002/hep.26195.

19. Gupta RA, Shah N, Wang KC, Kim J, Horlings HM, Wong DJ, Tsai MC, Hung T, Argani P, Rinn JL, Wang Y, Brzoska P, Kong B, et al. Long non-coding RNA HOTAIR reprograms chromatin state to promote cancer metastasis. Nature. 2010; 464:1071-6. https://doi.org/10.1038/ nature 08975 .
20. Du Y, Kong G, You X, Zhang S, Zhang T, Gao Y, Ye L, Zhang $\mathrm{X}$. Elevation of highly up-regulated in liver cancer (HULC) by hepatitis B virus X protein promotes hepatoma cell proliferation via down-regulating p18. J Biol Chem. 2012; 287:26302-11. https://doi.org/10.1074/jbc. M112.342113.

21. Purohit V, Rapaka R, Kwon OS, Song BJ. Roles of alcohol and tobacco exposure in the development of hepatocellular carcinoma. Life Sci. 2013; 92:3-9. https://doi.org/10.1016/j. lfs.2012.10.009.

22. Duan S, Mathews DH, Turner DH. Interpreting oligonucleotide microarray data to determine RNA secondary structure: application to the 3 ' end of Bombyx mori R2 RNA. Biochemistry. 2006; 45:9819 -32. https:// doi. org/10.1021/bi052618x.

23. Seitz HK, Stickel F. Risk factors and mechanisms of hepatocarcinogenesis with special emphasis on alcohol and oxidative stress. Biol Chem. 2006; 387:349-60. https://doi. org/10.1515/BC.2006.047.

24. Havens AM, Jung Y, Sun YX, Wang J, Shah RB, Buhring HJ, Pienta KJ, Taichman RS. The role of sialomucin CD164 (MGC-24v or endolyn) in prostate cancer metastasis. BMC Cancer. 2006; 6:195. https://doi.org/10.1186/1471-2407-6-195.

25. Tornesello ML, Buonaguro L, Tatangelo F, Botti G, Izzo F, Buonaguro FM. Mutations in TP53, CTNNB1 and PIK3CA genes in hepatocellular carcinoma associated with hepatitis $\mathrm{B}$ and hepatitis C virus infections. Genomics. 2013; 102:74-83. https://doi.org/10.1016/j.ygeno.2013.04.001.

26. Anwar SL, Krech T, Hasemeier B, Schipper E, Schweitzer N, Vogel A, Kreipe H, Lehmann U. Deregulation of RB1 expression by loss of imprinting in human hepatocellular carcinoma. J Pathol. 2014; 233:392-401. https://doi. org/10.1002/path.4376.

27. Puxbaum V, Nimmerfall E, Bauerl C, Taub N, Blaas PM, Wieser J, Mikula M, Mikulits W, Ng KM, Yeoh GC, Mach L. M6P/IGF2R modulates the invasiveness of liver cells via its capacity to bind mannose 6-phosphate residues. J Hepatol. 2012; 57:337-43. https://doi.org/10.1016/j.jhep.2012.03.026.

28. De Bac C, Stroffolini T, Gaeta GB, Taliani G, Giusti G. Pathogenic factors in cirrhosis with and without hepatocellular carcinoma: a multicenter Italian study. Hepatology. 1994; 20:1225-30.

29. Yu MW, You SL, Chang AS, Lu SN, Liaw YF, Chen CJ. Association between hepatitis $\mathrm{C}$ virus antibodies and hepatocellular carcinoma in Taiwan. Cancer Res. 1991; 51:5621-5.

30. Hassan MM, Hwang LY, Hatten CJ, Swaim M, Li D, Abbruzzese JL, Beasley P, Patt YZ. Risk factors for hepatocellular carcinoma: synergism of alcohol with viral hepatitis and diabetes mellitus. Hepatology. 2002; 36:120613. https://doi.org/10.1053/jhep.2002.36780.

31. Tanaka K, Hirohata T, Koga S, Sugimachi K, Kanematsu T, Ohryohji F, Nawata H, Ishibashi H, Maeda Y, Kiyokawa H, Tokunaga K, Irita Y, Takeshita Y, et al. Hepatitis C and 
hepatitis B in the etiology of hepatocellular carcinoma in the Japanese population. Cancer Res. 1991; 51:2842-7.

32. Forner A, Llovet JM, Bruix J. Hepatocellular carcinoma. Lancet. 2012; 379:1245-55. https://doi.org/10.1016/ S0140-6736(11)61347-0.

33. Singal A, Volk ML, Waljee A, Salgia R, Higgins P, Rogers MA, Marrero JA. Meta-analysis: surveillance with ultrasound for early-stage hepatocellular carcinoma in patients with cirrhosis. Aliment Pharmacol Ther. 2009; 30:37-47. https:// doi.org/10.1111/j.1365-2036.2009.04014.x.

34. Liu ZP. Identifying network-based biomarkers of complex diseases from high-throughput data. Biomark Med. 2016; 10:633-50. https://doi.org/10.2217/bmm-2015-0035.

35. El-Tawdi AH, Matboli M, El-Nakeep S, Azazy AE, AbdelRahman O. Association of long noncoding RNA and c-JUN expression in hepatocellular carcinoma. Expert Rev Gastroenterol Hepatol. 2016; 10:869-77. https://doi.org/10 $.1080 / 17474124.2016 .1193003$.

36. Wang K, Guo WX, Li N, Gao CF, Shi J, Tang YF, Shen F, Wu MC, Liu SR, Cheng SQ. Serum LncRNAs Profiles Serve as Novel Potential Biomarkers for the Diagnosis of HBV-Positive Hepatocellular Carcinoma. PLoS One. 2015; 10:e0144934. https://doi.org/10.1371/journal.pone. 0144934.

37. Kamel MM, Matboli M, Sallam M, Montasser IF, Saad AS, El-Tawdi AHF. Investigation of long noncoding RNAs expression profile as potential serum biomarkers in patients with hepatocellular carcinoma. Transl Res. 2016; 168:134-45. https://doi.org/10.1016/j.trsl.2015.10.002.

38. Quagliata L, Terracciano LM. Liver Diseases and Long Non-Coding RNAs: New Insight and Perspective. Front Med (Lausanne). 2014; 1:35. https://doi.org/10.3389/ fmed.2014.00035.

39. Quinlan AR, Hall IM. BEDTools: a flexible suite of utilities for comparing genomic features. Bioinformatics. 2010; 26:841-2. https://doi.org/10.1093/bioinformatics/btq033.

40. Volders PJ, Helsens K, Wang X, Menten B, Martens L, Gevaert K, Vandesompele J, Mestdagh P. LNCipedia: a database for annotated human lncRNA transcript sequences and structures. Nucleic Acids Res. 2013; 41:D246-51. https://doi.org/10.1093/nar/gks915.

41. Robinson MD, McCarthy DJ, Smyth GK. edgeR: a Bioconductor package for differential expression analysis of digital gene expression data. Bioinformatics. 2010; 26:13940. https://doi.org/10.1093/bioinformatics/btp616.

42. Duester G. A hypothetical mechanism for fetal alcohol syndrome involving ethanol inhibition of retinoic acid synthesis at the alcohol dehydrogenase step. Alcohol Clin Exp Res. 1991; 15:568-72.

43. Liu P, Xi Q, Ahmed A, Jaggar JH, Dopico AM. Essential role for smooth muscle BK channels in alcohol-induced cerebrovascular constriction. Proc Natl Acad Sci U S A. 2004; 101:18217-22. https://doi.org/10.1073/pnas.0406096102. 